\title{
BIBECHANA
}

A Multidisciplinary Journal of Science, Technology and Mathematics ISSN 2091-0762 (Print), 2382-5340 (0nline)

Journal homepage: http://nepjol.info/index.php/BIBECHANA

Publisher: Research Council of Science and Technology, Biratnagar, Nepal

\section{Molecular dynamics approach to the I431V mutational impact on thyroid hormone receptor-beta}

\author{
Tika Ram Lamichhane ${ }^{1}$, Sharma Paudel ${ }^{2}$, Binod Kumar Yadav², Hari Prasad Lamichhane ${ }^{{ }^{*}}$ \\ ${ }^{1}$ Central Department of Physics, Tribhuvan University, Kirtipur, Kathmandu, Nepal \\ ${ }^{2}$ Institute of Medicine, Tribhuvan University, Teaching Hospital, Maharajgunj, \\ Kathmandu, Nepal \\ *Email: hlamichhane1@gmail.com \\ Article history: Received 15 March, 2018; Accepted 16 September, 2018 \\ DOI: http://dx.doi.org/10.3126/bibechana.v13i0.21109 \\ This work is licensed under the Creative Commons CC BY-NC License.
}

https://creativecommons.org/licenses/by-nc/4.0/

\begin{abstract}
The point mutations like I431V on thyroid hormone receptor-beta (THR- $\beta$ ) gene cause resistance to thyroid hormones (RTH) with the clinical diagnosis of elevated free triiodothyronine (T3) and free thyroxin (T4) but not suppressed thyroid stimulating hormone (TSH) on the blood serum. Some ultrasonographic (USG) reports of the patients with RTH show thyroid gland disorder with goiter or nodule(s) or cyst(s) and some USG reports even with RTH are normal. I431V-mutant causes more steric hindrance while binding T3 into THR- $\beta$ than the native wild type THRT3. The residue on the 431-codon is dynamic in nature showing its flexibility over the course of entry and release of T3-hormone into/from the ligand binding pocket. The more increased solvent accessible surface area of I431V-mutant than that of native I431-residue makes the partial unfolding of the globular THR- $\beta$ protein. The smaller height of radial distribution function between I431-mutant and T3 shows the decrease in probability of finding the atomic particles nearby T3-hormone in THRT3-MT than in THRT3WT. The electrostatic interaction energy between native I431 and T3 is negative, but it is positive between I431V and T3. Moreover, the internal energy of I431V-mutant has been found smaller than that of native I431residue in THRT3 systems.
\end{abstract}

Keywords: Thyroid hormone receptor; resistance to thyroid hormones; point mutation; interaction energy; internal energy.

\section{Introduction}

Thyroid hormone receptors (THRs) are the nuclear receptor proteins that actively bind triiodothyronine (T3: hormone secreted by thyroid gland) to mediate the biological activities associated with gene transcription. THRs regulate gene expressions upon binding to the DNA sequences called as thyroid response elements (TREs). THRs have two isoforms THR- $\alpha$ and THR- $\beta$ that are in hetero-diversity in the different organs. T3 has the higher affinity to bind into both isoforms than thyroxin (T4). While T3 
binds to the ligand binding domain (LBD) of hydrophobicity, THR shows its structural change [1-4]. The carboxy-terminal activation domain forms an amphi-pathic helix $[2,3]$ as a part of cavity or LBD. The dynamic nature of helix-12 (H12 in Fig. 2-c) of THR is is important for the hormone binding, ligand dissociation, activation mechanism and regulation of transcription activity [5].

The mutational impact on THR reduces the hormone binding affinity and gene expression activity. Ultimately, the mutations leading to the resistance to thyroid hormone (RTH) cause the endocrine disorder. The inactivated THR- $\beta$ gene by means of homozygous mutant $\left(T h r b^{-/}\right)$in mice results in a goiter and elevated levels of thyroid hormones [6]. The mutant T327I found in THR- $\beta$ gene shows the paucity of symptoms and signs of thyroid dysfunction suggesting RTH despite the increased serum levels of T3, T4 and thyroid stimulating hormone (TSH) [7]. A rare mutation A268G found in axon 9 of THR- $\beta$ gene results RTH with elevated serum T3 and T4, TSH in the normal range and the episodic palpitations associated with mild dizziness [8]. A heterogeneous mutation G344R is identified in axon 10 of THR- $\beta$ gene of 10-month-old-female resulting in paucity of symptoms of RTH with normal T3, T4 and TSH levels [9]. Also, R383H mutation is found in THR- $\beta$ gene of a woman with inappropriate central secretion of TSH despite of elevated T3 and T4 [10]. The RTH patients are clinically euthyroid or hypothyroid depending on severity of mutation. A point mutation R429Q found in mice has the elevated serum thyroid hormones but the inappropriate TSH consistent with hypothalamic-pituitary RTH [11]. The active sites of these point mutations along with the site of I431V-mutant [12] in the THR- $\beta$ gene are indicated in Fig. (2-c). The helix-12 serves as a selective gatekeeper which is responsible for corepressor specificity by the wild type receptor (THR-WT). The point mutations in THR are all positioned to impact the gatekeeper functions and then to change corepressor specificity by the mutated receptor (THR-MT) [13, 14]. THR-WTs regulate normal physiological and developmental pathways, but THR-MTs result neoplastic and endocrine diseases.

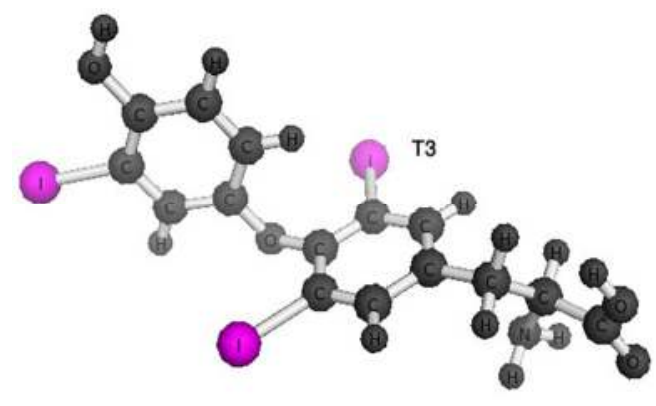

(a)

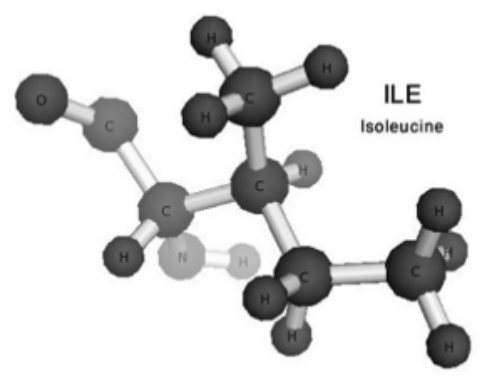

(b)

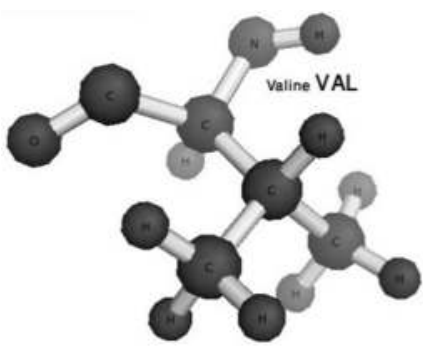

(c)

Fig. 1: Chemical structures of (a) triiodothyronine (T3): $\mathrm{C}_{15} \mathrm{H}_{12} \mathrm{I}_{3} \mathrm{NO}_{4}$ with molar mass $=650.98 \mathrm{~g} / \mathrm{mol}$, (b) isoleucine (ILE): $\mathrm{C}_{6} \mathrm{H}_{13} \mathrm{NO}_{2}$ with molar mass $=131.17 \mathrm{~g} / \mathrm{mol}$ and (c) valine (VAL): $\mathrm{C}_{5} \mathrm{H}_{11} \mathrm{NO}_{2}$ with molar mass $=117.15 \mathrm{~g} / \mathrm{mol}[$ prepared from PyMOL molecular graphics system].

The I431V point mutation is detected experimentally in THR- $\beta$ gene of a 14-year-old Brazilian girl with elevated T3 and T4 but unsuppressed TSH showing RTH with goiter [12]. This mutation occurs 
by replacing isoleucine (ILE) amino acid residue by valine (VAL) in 431 codon of THR- $\beta$ gene. I431V mutation exerts impairment of ligand-dependent release of corepressor, silencing mediator for retinoid and thyroid receptors (SMRT) with dominant negative effects on THR-WT and reducing potency. The chemical structures of T3, ILE and VAL are shown in Fig. 1. ILE is heavier and larger in size than VAL. T3 binding assay [12] shows that the hormone binding affinity of I431V-mutant THR- $\beta$ is 2.6 times lower than that in wild type THR- $\beta$. THR-WT, THRT3-WT and THRT3-MT show the distinct features reflecting in terms of root mean square deviation (RMSD), radius of gyration $\left(\mathrm{R}_{\mathrm{g}}\right)$, root mean square fluctuation (RMSF), IR spectra, radial distribution functions, solvent accessible surface area (SASA), interaction energy, internal energy, etc. The structural and physical properties of the wild type and the mutated protein-hormone systems can be studied by using molecular dynamics simulations (MDS).

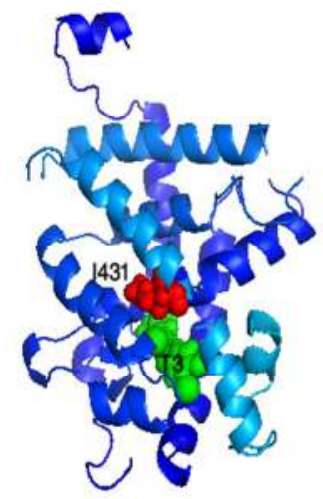

(a)

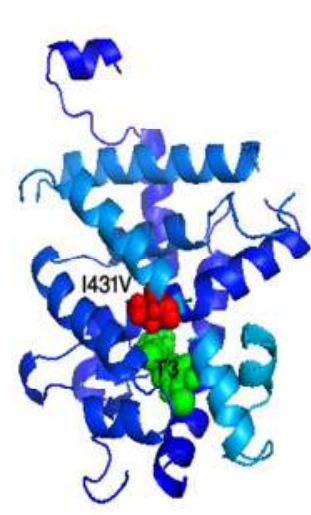

(b)

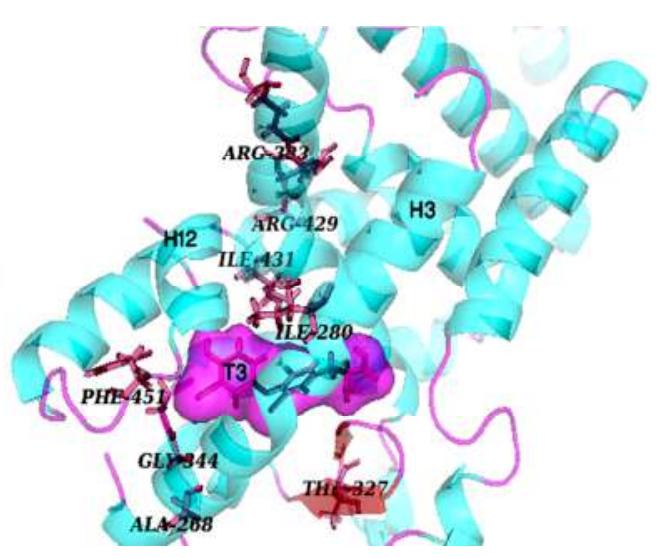

(c)

Fig.2: (a) Wild type THR- $\beta$ highlighting with I431 codon and T3 hormone, (b) I431V-mutant on THR- $\beta$ gene and (c) experimentally observed mutational sites on THR- $\beta$ gene [7-12]. The images are prepared from PyMOL molecular graphics system.

\section{Methodology}

The structure of wild type THRT3 was taken from the x-ray crystallographic data of protein data bank code-3GwS [15]. The CHARMM force field topologies and parameters [16, 17, 18, 19] were used for MDS of the protein-hormone systems. The hetero-atomic T3-hormone was parameterized first to describe its molecular geometry, atomic masses, charges and torsion barriers [19]. The generation of the complete systems of THR-WT, THRT3-WT and THRT3-MT starting from their protein structure files to the solvation in neutral water-ion environment, equilibration, production runs and analysis of the results were performed by using the interfaces of visual molecular dynamics (VMD) [20] and nanoscale molecular dynamics (NAMD) [21] computational packages.

From the wild type native structure of THRT3, the mutated THRT3 was made after the point mutation on 431-codon replacing ILE by VAL with the help of VMD-Mutagenesis. Unliganded THR-WT, T3liganded THRT3-WT and I431V-mutant THRT3-MT were solvated separately in the TIP3P water-box 
which was neutralized with $\mathrm{Na}^{+} \& \mathrm{Cl}^{-}$ions in the concentration of $0.15 \mathrm{~mol} / \mathrm{L}$. Each water-box has the periodic cell basis vectors 50.35, 61.92 and 74.78 Å along x, y and z-axes, respectively.

Configuration files were prepared by writing TCL-scripts to simulate the systems for which Langevin piston temperature, pressure and damping coefficient are $310 \mathrm{~K}, 1$ bar and $1 \mathrm{ps}^{-1}$, respectively. Oscillation period of the piston was $100 \mathrm{fs}$ with decay time of $50 \mathrm{fs}$ and periodic cell fluctuation was isotropic. The Langevin dynamics [22, 23] was applied for all atoms except hydrogens of the systems. Particle mesh Ewald (PME) was active with the grid dimensions of $(54,64,80 \AA)$ and the PME coefficient of 0.258 for the electrostatic force evaluation [24]. In adjusting the force field parameters used in MDS, 1-4 scaling was 1.0; cut-off, switching and pair-list distances for the electrostatic and van der Waals interactions were 12,10 and $14 \AA$, respectively. Finally, each system was geometrically optimized with 3000 conjugate gradient steps and then it was equilibrated up to $2 \mathrm{~ns}$ at $310 \mathrm{~K}$. To calculate the trajectory of atomic particles, the velocity Verlet algorithm [25] was used during MDS of the systems. All the simulations were performed adjusting integrator parameters of $2 \mathrm{fs} / \mathrm{step}$ constrained with rigid bonds of $\mathrm{H}$-atoms.

Conformational stability of the protein-hormone systems was tested by plotting RMSD, RG, RMSF and SASA of protein backbone, T3-hormone and residues of interest: I431 and I431V of THR-WT, THRT3WT and THRT3-MT. The dihedral angle distributions were analyzed from Ramachandran plots to test the steric hindrance among $\alpha$-helices, $\beta$-sheets and side chains and residues of the systems. The electrostatic and van der Waals interactions were observed between the native or mutated residue on 431-codon and T3-hormone by using NAMD-Energy-GUI. Also, number of H-bonding, probability density from radial distribution functions, IR spectral densities, internal energies of the native wild type and mutated systems were calculated, analyzed and compared by plotting the corresponding results over the simulation time.

\section{Result and Discussion}

THR- $\beta$ nuclear receptor isoform consists of a chain length of 259 amino acid residues. The protein structure of wild type THR- $\beta$ that we take for the simulation has total mass of $27640 \mathrm{amu}$ and 3895 atoms. The active ligand bound to the LBD of THR- $\beta$ is the thyroid hormone (T3) which has mass of 651 amu and 35 atoms. The plots of dihedral angles $(\phi, \psi)$, i. e. Ramachandran plots for THR-WT, THRT3WT and I431V mutant THRT3-MT are shown in Fig. 3. In these plots, most of the amino acid residues of parallel, anti-parallel and right twisted $\beta$-sheets lie in the blue region of I-quadrant; that of right handed $\alpha$ helix lie in the blue region of II-quadrant and left handed $\alpha$-helix lie in the green region of III-quadrant $[26,27]$. The residue GLY has no side chain so that it has no steric hindrance and it is allowed in the white region. The white region is sterically disallowed for all other amino acids except GLY. The blue regions are the most allowed regions for $\alpha$-helices and $\beta$-sheets. The green area is allowed for the shorter van der Waal radii of atoms and the related amino acids. By comparing three plots of Fig. 2, we observe that smaller number of amino acid residues lies in the disallowed region for THRT3-WT than for THRWT and THRT3-I431V. Thus, the larger steric hindrances occur for the unliganded and mutated THR- $\beta$. The clinical analysis shows that THRT3-WT is related to euthyroid or normal thyroid functions, THRWT is related to overt thyroidism and THRT3-MT is related to RTH [6-14]. 


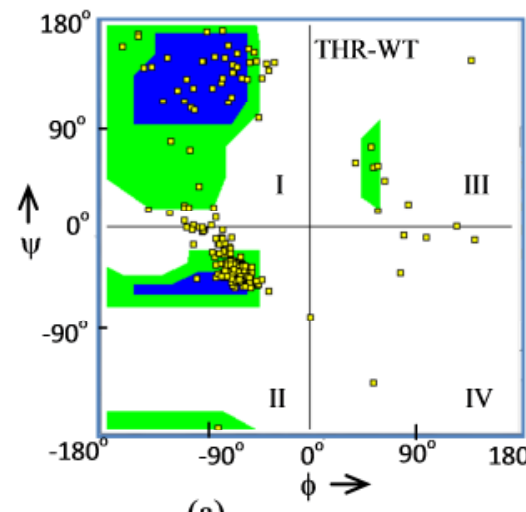

(a)

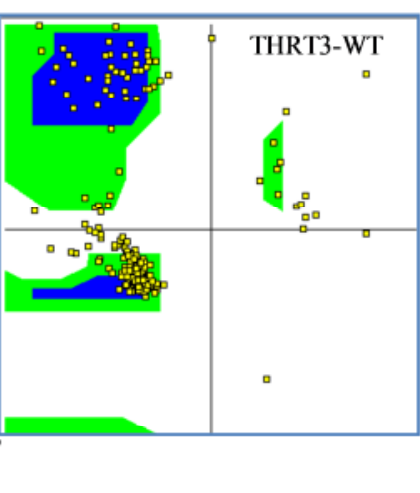

(b)

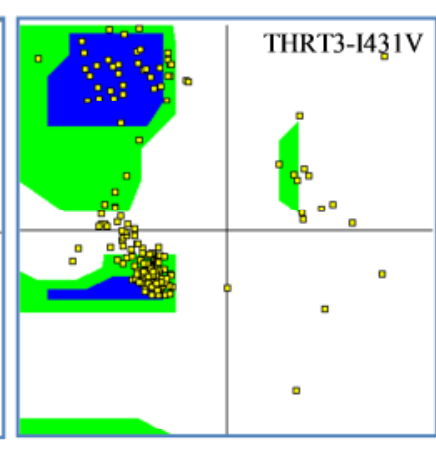

(c)

Fig. 3:Ramachandran plots for (a) wild type THR- $\beta$, (b) T3-liganded wild type THR- $\beta$ and (c) I431Vmutant THRT3.

The constancy of root mean square deviation (RMSD) and radius of gyration (RG) of the molecules THR$\beta$ protein and T3-hormone over the time frames of equilibration run (Fig. 4, 5) show the conformational stability and validity of force field topology and parameters used for MDS of wild type and mutated nuclear receptors. The more fluctuating RMSD and RG of residue ID-431 show its dynamic property. This dynamic property of the residues is significantly important while trapping T3 in and releasing T3 from the LBD of thyroid hormone receptors.

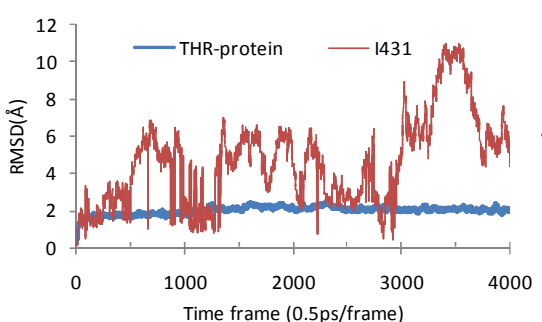

(a)

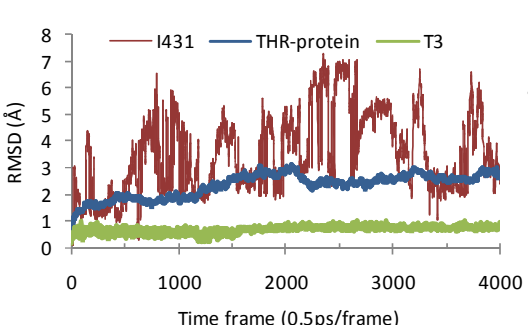

(c)

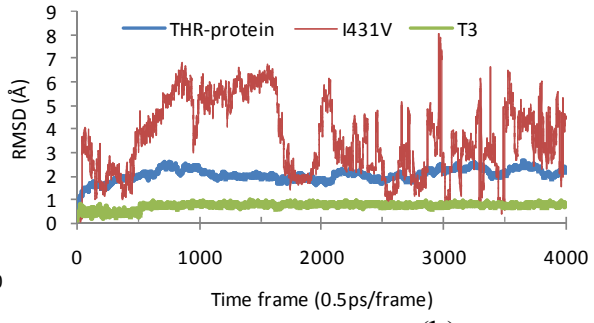

(b)

Fig.4: RMSD-plots of 431 residue, THR-protein and T3-hormone during equilibration of (a) wild type THR, (b) wild type THRT3 and (c) I431V-mutant THRT3.

The values of RMSD and RG of the molecules with the standard deviations of the data are listed in the Table 1. During their 2 ns equilibration run in neutral water-ion environment, RG of 431-residue of THR-WT is being smaller with larger RMSD, i.e. it is bending more towards the center of mass of the system than that of THRT3-WT and THRT3-MT. In other words, the nature of RMSD of 431-residue change from unliganded to liganded as well as wild type to mutated THR- $\beta$. The RMSD or RG of the protein is almost same with small fluctuations for these three cases. The protein RMSD is about $2 \AA$ and the protein RG is about $19 \AA$ for the wild type and the mutated THR- $\beta$ systems. The RMSD 
and RG of T3-hormone while bounded in the LBD of nuclear receptors are $0.7 \AA$ and $4.3 \AA$ respectively.

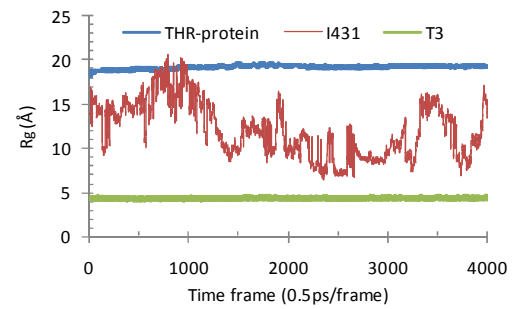

(a)

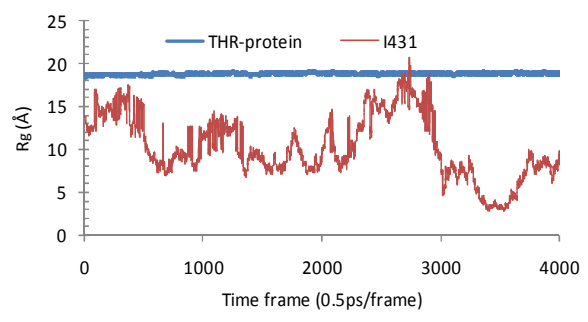

(b)

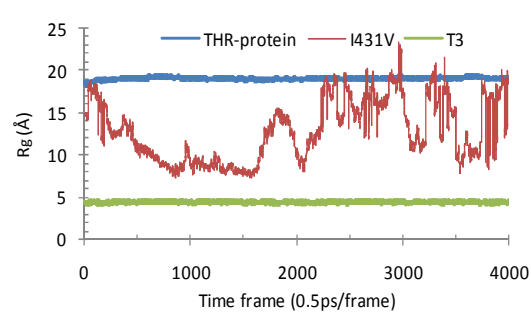

(c)

Fig. 5: RG-plots of 431 residue, THR-protein and T3-hormone during equilibration of (a) wild type THR, (b) wild type THRT3 and (c) I431V-mutant THRT3.

Table 1: Average values of root mean square deviation (RMSD) and radius of gyration (RG) of unliganded wild type and T3-liganded wild type and I431V-mutant THR- $\beta$ including with the standard deviation of the data obtained after MDS by their equilibration runs.

\begin{tabular}{|l|c|c|c|c|c|c|c|c|}
\hline \multirow{2}{*}{ Molecule } & \multicolumn{2}{|c|}{ THR-WT } & \multicolumn{3}{|c|}{ THRT3-WT } & \multicolumn{3}{c|}{ THRT3-MT } \\
\cline { 2 - 9 } & I431 & protein & I431 & T3 & protein & I431V & T3 & protein \\
\hline $\operatorname{RMSD}(\AA)$ & $4.6 \pm 2.4$ & $2.0 \pm 0.2$ & $3.6 \pm 1.6$ & $0.7 \pm 0.1$ & $2.0 \pm 0.3$ & $3.5 \pm 1.5$ & $0.7 \pm 0.1$ & $2.3 \pm 0.4$ \\
\hline $\operatorname{RG}(\AA)$ & $10.3 \pm 3.7$ & $18.8 \pm 0.1$ & $12.1 \pm 3.1$ & $4.3 \pm 0.1$ & $19.2 \pm 0.2$ & $12.8 \pm 3.6$ & $4.3 \pm 0.1$ & $19.1 \pm 0.1$ \\
\hline
\end{tabular}

In this study, the root mean square fluctuations (RMSF) of most of the residues of THRT3-WT are observed to be a little bit larger than that of THR-WT and THRT3-MT as shown in Fig. 6. In the I431V mutational site, the values of RMSF are $3.2 \AA$ for ILE of THR-WT, $4.1 \AA$ for ILE of THRT3-WT and 3.0 $\AA$ for VAL of THRT3-MT. The residues in helix-1 have the highest RMSF so that the helix-1in THRT3WT is more flexible.

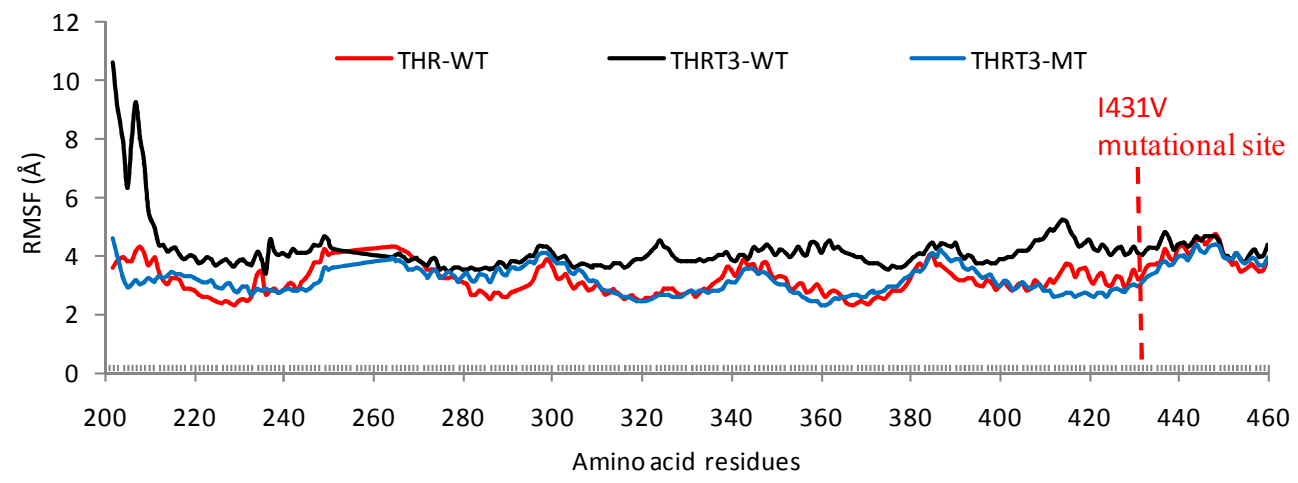

Fig. 6: RMSF of amino acid residues of THR-WT, THRT3-WT and I431V-mutant THRT3-MT during their 2 ns equilibration run. 
Solvent accessible surface area (SASA) of THR-WT, THRT3-WT and THRT3-MT are observed to be slightly different in the extension radius of $1.4 \AA$. After 500 frames of energy minimization and equilibration, the SASA with the standard deviation of the data from frame to frame simulation up to $2 \mathrm{~ns}$ is $13620.1 \pm 253.07 \AA^{2}$ for THR-WT, $13952.4 \pm 130.9 \AA^{2}$ for THRT3-WT and $13721.0 \pm 147.9 \AA^{2}$ for THRT3-MT. In this way, the SASA of 431-residue is $14.8 \pm 9.0 \AA^{2}$ for THR-WT, $12.6 \pm 10.1 \AA^{2}$ for THRT3-WT and 17.4 $\pm 8.1 \AA^{2}$ for THRT3-MT. The lower SASA indicates the higher thermodynamic stability of the protein. The SASA of T3-hormone is observed to be zero indicating the hydrophobicity of LBD of THR- $\beta$. The mutation on the solvent accessible residue causes the conformational change by partial unfolding of the protein with increasing SASA [28]. Such effect is more intensive if the mutation occurs on the surface residue than on the interior of the protein. The I431V mutation is the interior one so that no significant change in SASA of wild type and mutated THR- $\beta$ has been observed after their $2 \mathrm{~ns}$ equilibration. However, the total SASA of THR-WT and THRT3-MT are more fluctuating than that of THRT3-WT up to 3000 frames of the equilibration runs (Fig. 7-a). Furthermore, SASA of I431V-mutant is higher and less fluctuating than that of native and wild type I431-rsidue of THR- $\beta$ (Fig.7-b).

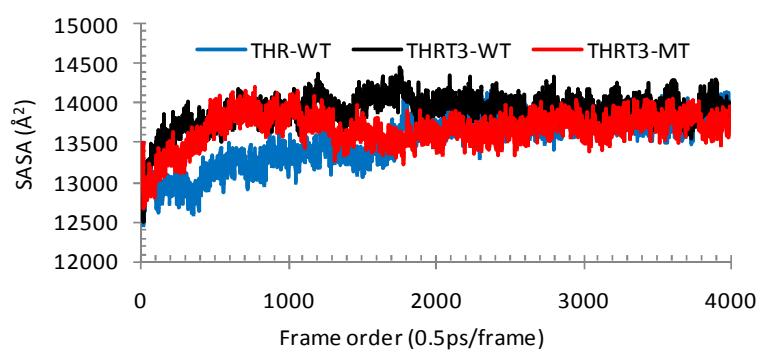

(a)

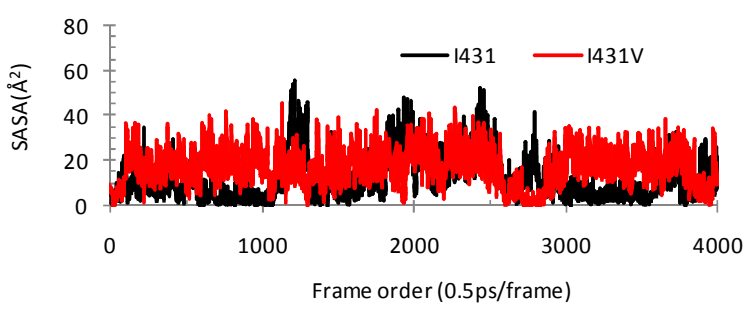

(b)

Fig. 7: (a) SASA of protein in wild type THR, THRT3 and mutated-THRT3, (b) SASA of I431 residue in THRT3-WT and I431V mutant in THRT3-MT during their equilibration runs.

The distributions of number of H-bonds along with the frame order of the simulations up to 2 ns equilibrations are shown in Fig. 8. Here, the average values of $\mathrm{H}$-bond number with the standard deviation of the data after 500 frames with the donor-acceptor distance $4.0 \AA$ and cut-off angle $40^{\circ}$ among $\mathrm{N}$ and $\mathrm{O}$ atoms are $5 \pm 2$ for I431-THR(WT), $16 \pm 4$ for T3-THR(WT), $4 \pm 2$ for I431-THR(MT) and $15 \pm$ 4 for T3-THR(MT).

During MDS of the wild type and mutated THR- $\beta$ systems, the distance between I431 and T3 in THRT3WT varies as $10.2 \pm 2.6 \AA$ and the distance between I431V and T3 in THRT3-MT varies as $13.1 \pm 2.0 \AA$ as shown in Fig. 9-b. Such distance is smaller and more fluctuating in THRT3-WT than in THRT3-MT. This is one of the indicators of T3 resistant features of mutated nuclear receptor proteins that cause RTH.

Radial distribution function [29] or pair auto-correlation function $\mathrm{g}(\mathrm{r})$ in a system of particles measures probability of finding a particle at $r$ distance away from the reference particle. The distribution function $\mathrm{g}(\mathrm{r})$ between I431-residue and T3-hormone has the higher value than that between I431V and T3 
and it lies in the range of $5 \AA$ and $18 \AA$ as shown in Fig. 9-c. In other words, the number density of mutated residue is less than that of the native wild type residue to respond for T3-hormone and TREs.

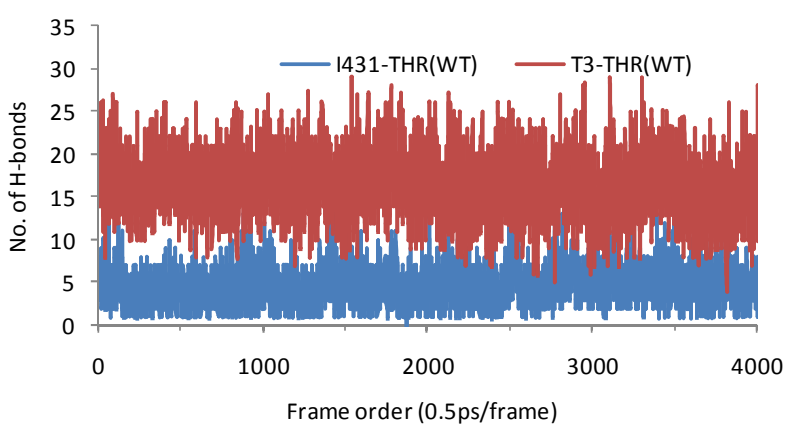

(a)

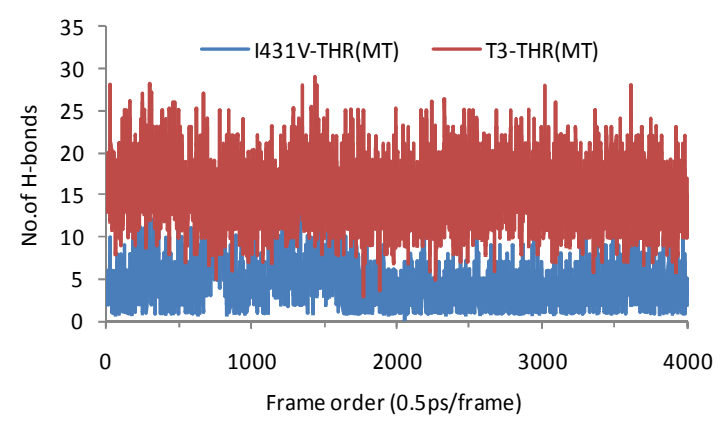

(b)

Fig. 8: Variation of number of H-bonds between 431-residue and protein vs. simulation time in case of (a) wild type and (b) I431V-mutant THRT3 systems.

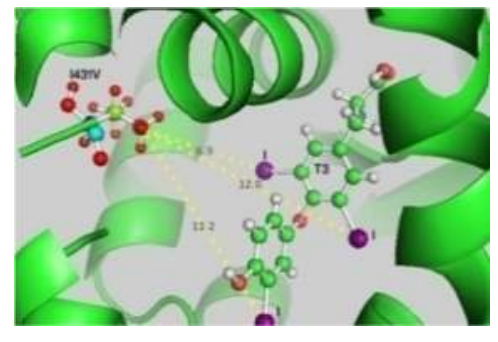

(a)

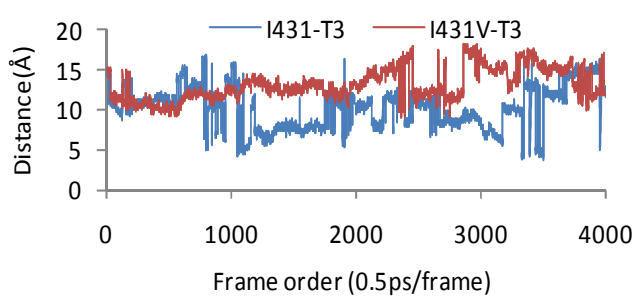

(b)

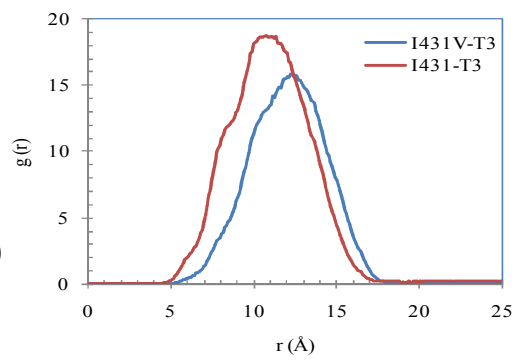

(c)

Fig. 9: (a) Positions of I431V-mutant and T3-hormone in THRT3-MT, (b) changing distance between 431-codon and T3 while equilibrating THRT3-WT and THRT3-MT systems and (c) radial distribution functions between 431-residue and T3-hormone in case of wild type and mutated THRT3.

IR spectral densities using total dipole moment of the atoms in 431-residue and $\mathrm{T} 3$ are calculated in the frequency range of 0 to $2000 \mathrm{~cm}^{-1}$ and their plots are different for THRT3-WT and THRT3-MT as shown in Fig. 10. The maximum IR-intensity of T3 in the wild type receptor is 0.041 at $1837.42 \mathrm{~cm}^{-1}$ and that of I431 (wild type) is 0.027 at $1637.88 \mathrm{~cm}^{-1}$. Again, the maximum IR-intensity of T3 in the mutated receptor is 0.037 at $1313.63 \mathrm{~cm}^{-1}$ and that for $\mathrm{I} 431 \mathrm{~V}$-mutant is 0.029 at $1571.37 \mathrm{~cm}^{-1}$. The distribution has larger number of high intensity peaks for T3 and I431V in THRT3-MT than in THRT3-WT.

Total non-bonding interaction energy is the sum of electrostatic and van der Waal's interaction energy. The changing values of interaction energy between 431-residue and T3-hormone in wild type and mutated THR- $\beta$ systems are plotted with respect to the frame order in Fig. 11 and these are averaged 
over the time frames from 500 to 4013. The average values of electrostatic and van der Waals interaction energy (from NAMD-Energy-GUI using switching and cut-off distances of $10 \AA$ and $12 \AA$, respectively) with their standard deviations are $-0.25 \pm 0.17 \mathrm{kcal} / \mathrm{mol}$ and $-0.18 \pm 0.07 \mathrm{kcal} / \mathrm{mol}$ between I431 and T3 of THRT3-WT and $0.21 \pm 0.08 \mathrm{kcal} / \mathrm{mol}$ and $-0.07 \pm 0.05 \mathrm{kcal} / \mathrm{mol}$ between I431V and T3 of THRT3MT, respectively. Thus, the electrostatic energy between I431 and T3 in THRT3-WT is negative and more fluctuating and that between I431V and T3 in THRT3-MT is positive and less fluctuating over the simulation time.

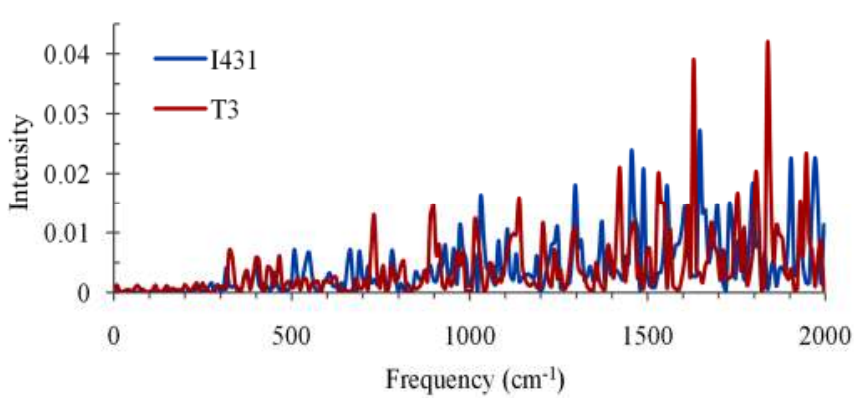

(a)

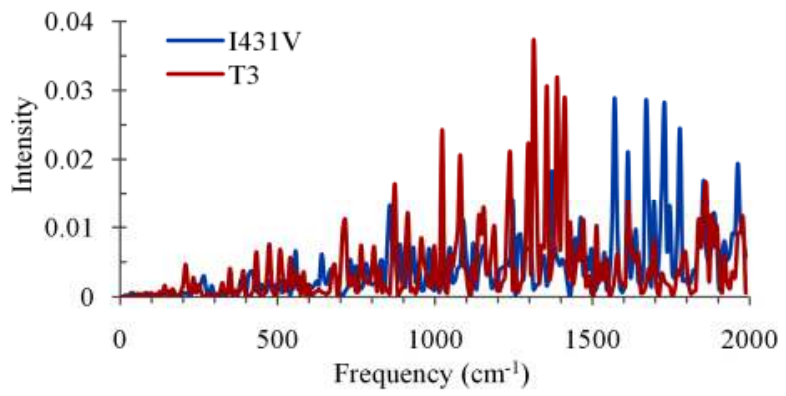

(b)

Fig. 10: IR spectra of (a) I431 residue and T3-hormone in wild type THRT3 and (b) I431V-mutant and T3-hormone in mutated THRT3.

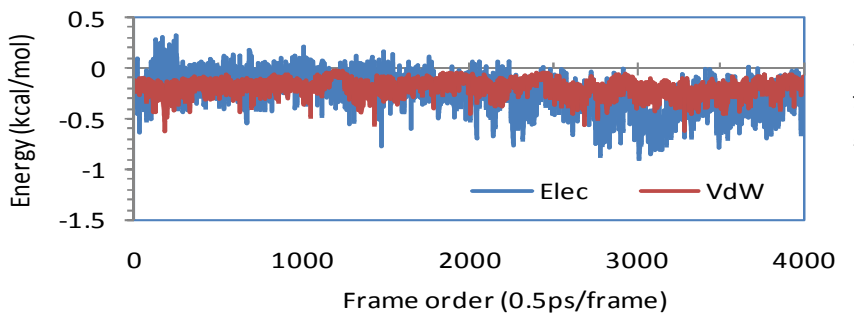

(a)

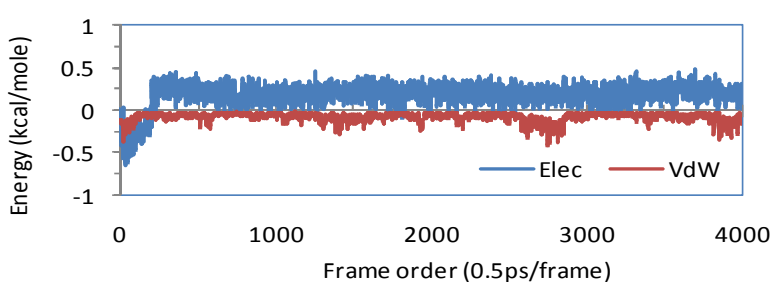

(b)

Fig. 11: Electrostatic and van der Waals interaction energies (a) between $I 431$ and T3 in wild type THRT3 and (b) between I431V and T3 in mutated THRT3.

Internal energy of the biomolecular system is the sum of bonding and non-bonding potentials. Coulomb energy is the largest and its fluctuation during SMD makes the total internal energy fluctuate about its mean value. Using NAMD-Energy-GUI with switching and cut-off distances of $10 \AA$ and $12 \AA$, the internal energy of 431-residue, bounded T3-hormone and protein of THR-WT, THRT3-WT and THRT3MT are calculated and the average values for the corresponding systems are listed in the Table 2 . The variations of the related internal energy with respect to the simulation time are plotted in the Fig. 12. The plots for the individual potential terms show that the major contributor of fluctuations in the internal energy is the electrostatic energy of the related system. 
Table 2: Internal energy of the components of unliganded wild type, T3-liganded wild type and I431Vmutant THR- $\beta$ systems averaged over the values from the last $0.25 \mathrm{~ns}$ of their equilibrations where the including error represents standard deviation of the data.

\begin{tabular}{|l|c|c|c|c|c|c|c|c|}
\hline \multirow{2}{*}{ Molecule } & \multicolumn{2}{|c|}{ THR-WT } & \multicolumn{3}{|c|}{ THRT3-WT } & \multicolumn{3}{c|}{ THRT3-MT } \\
\cline { 2 - 9 } & $\mathrm{I} 431$ & protein & $\mathrm{I} 431$ & $\mathrm{~T} 3$ & protein & I431V & T3 & protein \\
\hline Int. energy & 42.82 & -822.38 & 42.88 & 128.73 & -644.21 & $36.13 \pm$ & 124.41 & -756.54 \\
(kcal/mol) & \pm 3.21 & \pm 67.67 & \pm 3.18 & \pm 4.52 & \pm 109.87 & 3.07 & \pm 4.63 & \pm 104.22 \\
\hline
\end{tabular}

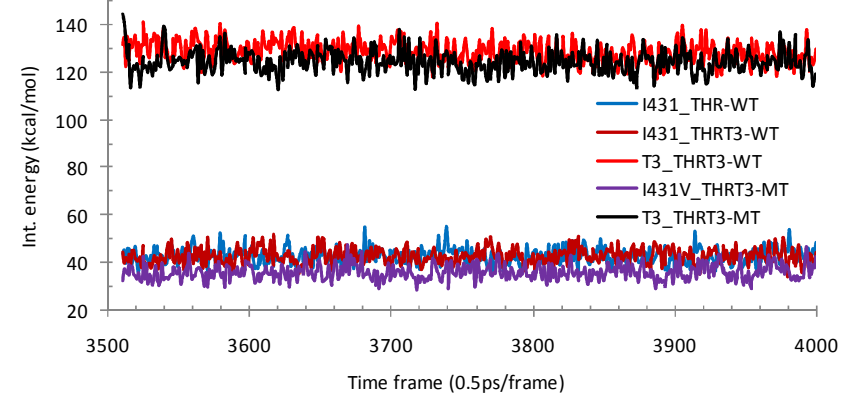

(a)

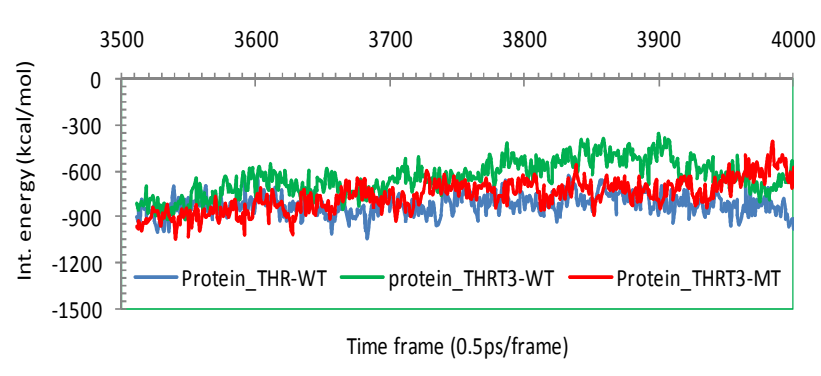

(b)

Fig. 12: Fluctuating total internal energy along the frame order of (a) I431-residue and T3, and (b) THRproteins for unliganded as well as T3-liganded wild type and I431V-muatant THR- $\beta$ systems in the last $0.25 \mathrm{~ns}$ of their equilibrations.

\section{Conclusion}

Conformational properties and interactions of mutational residues and T3-hormone of unliganded and T3liganded thyroid hormone receptor-beta $($ THR- $\beta$ ) can be studied by means of molecular dynamics simulations (MDS). The almost constant values of root mean square deviation (RMSD $\approx 2 \AA$ ) and radius of gyration $(\mathrm{RG} \approx 19 \AA$ ) of native and I431-mutant THR- $\beta$ proteins show their conformational stability during MDS. The native I431-residue has largely fluctuating RMSD and RG showing its dynamic property in adapting and releasing T3-hormone. The experimentally verified I431V mutation on THR- $\beta$ gene (Ferreira et al., 2010) is the subject of this computational study. This mutation causes resistance to thyroid hormones [12]. Solvent accessible surface area (SASA) is larger for the I431V-mutant indicating partial unfolding of the globular protein. However, the root mean square fluctuation of I431V-mutant is smaller in comparison with that of native wild type I431. The SASA of T3 is zero verifying the hydrophobicity upon binding to the ligand binding domain (LBD) of THR- $\beta$.

Comparing the radial distribution functions $\mathrm{g}(\mathrm{r})$ for the atomic particles between I431 and T3 as well as I431V and T3, the probability density is higher in the former wild type than the latter mutated type for which $g(r)$ covers the distance of $5 \AA$ to $18 \AA$. IR spectral density calculations for I431-residue and T3hormone in the frequency range of $0-2000 \mathrm{~cm}^{-1}$ imply that greater number of intensity maxima is found in THRT3-MT than in THRT3-WT. This signifies higher dipole moment distributions by supporting less globular stability of the mutated THR- $\beta$ than that of the wild type THR- $\beta$. Furthermore, electrostatic interaction energy between I431V-mutant and T3 is positive whereas it is negative between 
native I431-residue and T3. In this study, internal energy of the native I431-residue is $42.88 \pm 3.18$ $\mathrm{kcal} / \mathrm{mol}$ and that of wild type I431V-mutant is $36.13 \pm 3.07 \mathrm{kcal} / \mathrm{mol}$ in THRT3 systems. The major contributor of such change in the internal energy is the electrostatic energy.

\section{Acknowledgement}

This work was partially supported by Nepal Academy of Science and Technology (NAST) as the Ph. D. fellowship grant to the first author. The computing facility was provided by Central Department of Physics, Tribhuvan University, Kathmandu, Nepal.

\section{References}

[1] L. Martínez, I. Polikarpov, M. S. Skaf, Only subtle protein conformational adaptations are required for ligand binding to thyroid hormone receptors: simulations using a novel multipoint steered molecular dynamics approach,J. Phys. Chem. B 112 (2008) 10741-10751. doi.org/10.1021/jp803403c.

[2] L. Martínez, P. C. T. Souza, W. Garcia, F. A. Batista, R. V. Portugal, A. S. Nascimento, M. Nakahira, L. M. Lima, I. Polikarpov, M. S. Skaf, On the denaturation mechanisms of the ligand binding domain of thyroid hormone receptors, J. Phys. Chem. B 114 (2010) 1529-1540. doi.org/ 10.1021/jp911554p.

[3] R. L. Wagner, J. W. Apriletti, M. E. McGrath, B. L. West, J. D. Baxter, R. J. Fletterick, A structural role for hormone in the thyroid hormone receptor, Nature 378 (1995) 690-697.doi.org/0.1038/378690a0.

[4] R. V. Weatherman, R. J. Fletterick, T. S. Scanlan, Nuclear-receptor ligands and ligand-binding domains, Annu. Rev. Biochem. 68 (1999) 559-581.doi.org/10.1146/annurev.biochem.68.1.559.

[5] B. C. Kallenberger, J. D. Love, V. K. Chatterjee, J. W. Schwabe, A dynamic mechanism of nuclear receptor activation and its perturbation in a human disease, Nat. Struct. Biol.10 (2003) 136140.doi.org/10.1038/nsb892.

[6] D. Forrest, E. Hanebuth, R. J. Smeyne, N. Everds, C. L. Stewart, J. M. Wehner, T. Curran, Recessive resistance to thyroid hormone in mice lacking thyroid hormone receptor beta: evidence for tissue-specific modulation of receptor function, EMBO J. 15 (1996) 3006-3015.doi.org/10.1002/j.14602075.1996.tb00664.X.

[7] J. H. Lee, E. Y. Kim, Resistance to thyroid hormone due to a novel mutation of thyroid hormone receptor beta gene, Ann. Pediatr. Endocrinol. Metab. 19 (2014) 229-231. doi.org/10.6065/apem.2014.19.4.229.

[8] K. El Shafie, A. Ouhtit, Y. Al Farsi, A. Al Sayegh, M. Al Shafaee, A rare thyroid hormone receptor beta (THR $\beta)$ gene mutation in a 15-year-old girl with thyroid hormone resistance syndrome: a case report, J. Med. Case Rep. 8 (2014) 12. doi.org/ 10.1186/1752-1947-8-12.

[9] W. Yang, J. Yan, Y. Sang, Resistance to thyroid hormone caused by a G344R mutation of thyroid hormone receptor beta gene: A case report study,Endocrinol. Metab.Synd. 5 (2016) 231.doi.org/10.4172/2161$\underline{1017.1000231 .}$

[10] J. D. Safer, M. G. O'connor, S. D. Colan, S. Srinivasan, S. R. Tollin, F. E. Wondisford, The thyroid hormone receptor- $\beta$ gene mutation $\mathrm{R} 383 \mathrm{H}$ is associated with isolated central resistance to thyroid hormone, J. Clin. Endocrinol.Metab. 84 (1999) 3099-3109.doi.org/10.1210/jcem.84.9.5985.

[11] D. S. Machado, A. Sabet, L. A. Santiago, A. R. Sidhaye, M. I. Chiamolera, T. M. Ortiga-Carvalho, F. E. Wondisford, A thyroid hormone receptor mutation that dissociates thyroid hormone regulation of gene expression in vivo, Pro. Natl. Acad. Sci. 106 (2009) 9441-9446.doi.org/10.1073/pnas.0903227106. 
[12] M. Ferreira Azevedo, G. B. Barra, L. D. D. Medeiros, L. A. Simeoni, L. A. Naves, F. D. A. R. Neves, A novel mutation of thyroid hormone receptor beta (I431V) impairs corepressor release, and induces thyroid hormone resistance syndrome, Arq. Bras.Endocrinol.Metab. 52 (2008) 1304-1312. doi.org/10.1590/S000427302008000800016.

[13] M. D. Rosen, M. L. Privalsky, Thyroid hormone receptor mutations in cancer and resistance to thyroid hormone: perspective and prognosis, J. Thyroid Research, 2011 (2011) 20.doi.org/10.4061/2011/361304.

[14] M. D. Rosen, M. L. Privalsky,Thyroid hormone receptor mutations found in renal clear cell carcinomas alter corepressor release and reveal helix 12 as key determinant of corepressor specificity, Mol. Endocrinol. 23 (2009) 1183-1192. doi.org/10.1210/me.2009-0126.

[15] A.S. Nascimento, S. M. G. Dias, F. M. Nunes, R. Aparício, A. L. Ambrosio, Bleicher, L., A. C. M. Figueira, M. A. M. Santos, M. de Oliveira Neto, H. Fischer, M. Togashi, Structural rearrangements in the thyroid hormone receptor hinge domain and their putative role in the receptor function, J. Mol. Biol. 360 (2006) 586598.doi.org/10.1016/j.jmb.2006.05.008.

[16] A. D. MacKerell, M. Feig, C. L. Brooks, Extending the treatment of backbone energetics in protein force fields: Limitations of gas-phase quantum mechanics in reproducing protein conformational distributions in molecular dynamics simulations, J. Comp. Chem. 25(2004) 1400-1415. doi.org/10.1002/jcc.20065.

[17] A. D. MacKerell, D. Bashford, M. Bellott, R. L. Dunbrack, J. D. Evanseck, M. J. Field, S. Fischer, J. Gao, H. Guo, S. Ha, D. Joseph-McCarthy, All-atom empirical potential for molecular modeling and dynamics studies of proteins, J. Phys. Chem. B 102 (1998) 3586-3616. doi.org/10.1021/jp973084f.

[18] W. L. Jorgensen, J. Chandrasekhar, J. D. Madura, R. W. Impey, M. L. Klein, Comparison of simple potential functions for simulating liquid water, J. Chem. Phys. 79 (1983) 926-935. doi.org/10.1063/1.445869.

[19] V. Zoete, M. A. Cuendet, A. Grosdidier, O. Michielin, SwissParam: a fast force field generation tool for small organic molecules, J. Comp. Chem.32(2011) 2359-2368. doi.org/ 10.1002/jcc.21816.

[20] W. Humphrey, A. Dalke, K. Schulten, VMD—Visual Molecular Dynamics, J. Mol. Graphics 14 (1996) 3338. doi.org/10.1016/0263-7855(96)00018-5.

[21] J. C. Phillips, R. Braun, W. Wang, J. Gumbart, E. Takhorshid, E. Villa, C. Chipot, R. D. Skeel, L. Kale, K. Schulten,Scalable molecular dynamics with NAMD, J. Comput. Chem. 26 (2005) 1781-1802. doi.org/ $10.1002 / j c c .20289$.

[22] S. E. Feller, Y. Zhang, R. W. Pastor, B. R. Brooks, Constant pressure molecular dynamics simulation: the Langevin piston method, J. Chem. Phys. 103 (1995) 4613-4621.doi.org/10.1063/1.470648.

[23] G. Bussi, D. Donadio, M. Parrinello, Canonical sampling through velocity rescaling, J. Chem Phys. 126(2007) 014101.doi.org/10.1063/1.2408420.

[24] T. Darden, D. York, L. Pedersen, Particle mesh Ewald: an $N \cdot \log (\mathrm{N})$ method for Ewald sums in large systems, J. Chem. Phys. 98 (1993) 10089-10092. doi.org/10.1063/1.464397.

[25] L. Verlet, Computer "experiments" on classical fluids. I. Thermodynamical properties of Lennard-Jones molecules, Phy. Rev. 159 (1967) 98.doi.org/10.1103/PhysRev.159.98.

[26] S. A. Hollingsworth, P. A. Karplus, A fresh look at the Ramachandran plot and the occurrence of standard structures in proteins, Biomol. concepts 1(2010) 271-283. doi.org/10.1515/bmc.2010.022.

[27] B. K. Ho, R. Brasseur, The Ramachandran plots of glycine and pre-proline, BMC Struct. Biol. 5 (2005) 14.doi.org/10.1186/1472-6807-5-14.

[28] D. Gilis, M. Rooman, Stability changes upon mutation of solvent-accessible residues in proteins evaluated by database-derived potentials, J. Mol. Biol. 257(1996) 1112-1126. doi.org/10.1006/jmbi.1996.0226. 
Tika Ram Lamichhane et al./ BIBECHANA 16 (2019) 79-91 : RCOST p.91 (Online Publication: Dec., 2018)

[29] M. Fernández, J. Caballero, L. Fernández, J. I. Abreu, M. Garriga, Protein radial distribution function (PRDF) and Bayesian-Regularized Genetic Neural Networks for modeling protein conformational stability: Chymotrypsin inhibitor 2 mutants, J. Mol. Graph. Modl. 26(2007) 748-759. doi.org/10.1016/j.jmgm.2007.04.011. 